\title{
Emerged Metallicity in Molecular Ferromagnetic Wires
}

Yulong Huang, ${ }^{\dagger, \# ~ T r a v i s ~ M i t c h e l l, ~}{ }^{\S} \#$ Dillon C. Yost, ${ }^{\ddagger}$ Yong Hu, ${ }^{\dagger}$ Jason B. Benedict, ${ }^{\S}$ Jeffrey C.

Grossman, ${ }^{\ddagger}$ Shenqiang $\operatorname{Ren}^{\dagger}, \stackrel{,}{,}, *$

†Department of Mechanical and Aerospace Engineering, University at Buffalo, The State University of New York, Buffalo, NY, 14260, USA

${ }^{\S}$ Department of Chemistry, University at Buffalo, The State University of New York, Buffalo, NY, 14260, USA

tDepartment of Materials Science and Engineering, Massachusetts Institute of Technology, Cambridge, MA 02139, USA

${ }^{\perp}$ Research and Education in energy, Environment and Water (RENEW) Institute, University at Buffalo, The State University of New York, Buffalo, NY, 14260, USA.

*E-mail: shenren@buffalo.edu

\#These authors contributed equally to this work. 
This PDF file includes:

Section 1. Sample synthesis

Section 2. Structural and morphologic characterizations

Section 3. Single Crystal X-ray Diffraction

Section 4. Magnetic susceptibility and hysteresis loops

Section 5. Electrical transport properties

Section 6. First-principles computation

Section 7. Spectroscopic properties

Section 8. Diamagnetic transition

SI References

Other supporting materials for this manuscript include the following:

Data S1. Crystallographic file. 


\section{Materials and Methods}

Synthesis of $\mathbf{P P h}_{4} \mathbf{N}(\mathbf{C N})_{2} .8 .386 \mathrm{~g}$ tetraphenylphosphonium bromide (TCI, $\mathrm{PPh}_{4} \mathrm{Br},>98.0 \%$ ) was dissolved into $500 \mathrm{ml}$ distilled water by stirring for one hour, then $15 \mathrm{ml}$ aqueous sodium dicyanamide $\left(1.781 \mathrm{~g} \mathrm{NaN}(\mathrm{CN})_{2}\right.$, TCI, $\left.>98.0 \%\right)$ solution was added to produce shining white $\mathrm{PPh}_{4} \mathrm{~N}(\mathrm{CN})_{2}$ precipitate. About $7.3 \mathrm{~g} \mathrm{PPh}_{4} \mathrm{~N}(\mathrm{CN})_{2}$ product was collected for the following electrolyte preparation.

Single-crystal wire growth. Semiconducting $(\mathrm{ET}) \mathrm{Cu}\left[\mathrm{N}(\mathrm{CN})_{2}\right]_{2}$ wires were grown using a customized electrocrystallization method based on literatures ${ }^{1}$. First, $0.1 \mathrm{mmol} \mathrm{CuCl}$ powder (Sigma-Aldrich, 97\%) and $0.1 \mathrm{mmol} \mathrm{PPh}_{4} \mathrm{~N}(\mathrm{CN})_{2}$ were mixed together in $12 \mathrm{ml} \mathrm{1,1,2-}$ trichloroethane (Sigma-Aldrich, 97\%, TCA) solution with 10\% ethanol for 2-hour stirring and 20-minute ultrasonication. Then, $0.05 \mathrm{mmol}$ bis(ethylenedithio)tetrathiafulvalene (TCI, ET) was added into the solution and was stirred for 2 hours and put for ultrasonication for 20 minutes. The undissolved chemicals were filtered to get a clean light-yellow solution that was transferred as electrolyte into a $20 \mathrm{ml}$ glass bottle. A platinum wire electrode with a thickness of $0.5 \mathrm{~mm}$ was used as the cathode, while a fluorine doped tin oxide (FTO) substrate (about 2 $\mathrm{cm}^{2}$ was immerged into the electrolyte) was regarded as the anode. Two holes were made on the cap of the glass bottle to let the Pt electrode and FTO substrate go through and get merged into solution. The glass bottle was sealed well using a glue gun. The electrochemical cell was applied an increasing current by Keithley 2400 that was slowly increased from $0.1 \mathrm{uA}$ to 0.9 $\mathrm{uA}$ in one month. Finally, the FTO substrate was covered by $(\mathrm{ET}) \mathrm{Cu}\left[\mathrm{N}(\mathrm{CN})_{2}\right]_{2}$ wires on the conducting side. Here a constant low current $(0.1 \sim 0.2 \mathrm{uA})$ is avoided so that kinetic process promotes the wire growth.

Structural and morphologic characterizations: Powder X-ray diffraction on $(\mathrm{ET}) \mathrm{Cu}\left[\mathrm{N}(\mathrm{CN})_{2}\right]_{2}$ wires with an FTO substrate was conducted on the Rigaku Ultima IV (40 $\mathrm{kV}, 44 \mathrm{~mA}, \mathrm{Cu} \mathrm{K \alpha}$ ) from $5^{\circ}$ to $80^{\circ}$ by a scanning speed of 0.5 degree $/ \mathrm{min}$. The topological morphology of $(\mathrm{ET}) \mathrm{Cu}\left[\mathrm{N}(\mathrm{CN})_{2}\right]_{2}$ wires was measured by Field Emission Scanning Electron Microscope (FESEM) Carl Zeiss AURIGA $(200 \mathrm{kV})$. The elements in $(\mathrm{ET}) \mathrm{Cu}\left[\mathrm{N}(\mathrm{CN})_{2}\right]_{2}$ wires were detected by Oxford Energy-dispersive X-ray Spectrometer (EDS). High resolution transmission electron microscopic images and selected electron diffraction patterns were taken on JEOL JEM 2010. 
Single-Crystal X-ray Diffraction Methods: A single crystal suitable for X-ray diffraction was mounted on the tip of a glass fiber with oil and placed on a Bruker SMART APEX II CCD diffractometer installed at a rotating anode source (Mo-K $\alpha$ radiation, $\lambda=0.71073 \AA$ ) with a detector distance of $40.00 \mathrm{~mm}$ from the crystal and a $2 \theta$-angle of $-25^{\circ}$. A total of 1800 frames were collected using five $180^{\circ} \omega$-scans $\left(0.5^{\circ}\right.$ scan width) at different $\varphi$-angles $\left(\varphi=0^{\circ}\right.$ to $288^{\circ}$ in $72^{\circ}$ increments), nominally covering complete reciprocal space. Data reduction was completed using SAINT version 8.40A, and a multi-scan absorption correction was applied using SADABS version 2016 included in the Bruker APEX32 software suite. Space-group determination was performed using the XPREP utility included in the SHELXTL ${ }^{3}$ software package. Using Olex2 ${ }^{4}$, the structure was solved with ShelXT ${ }^{5}$ using intrinsic phasing and refined with ShelXL ${ }^{6}$ using least squares minimization (full-matrix least-squares on $\mathrm{F}^{2}$ ).

Spectroscopy measurements: Raman spectroscopy measurements were carried on Renishaw inVia Raman Microscope with an excitation wavelength of $514 \mathrm{~nm}$. Wire sample was sealed into a small vaccum chamber where temperature of sample stage is stabilized by a cryogenic programmable intelligent temperature controller (MercuryiTC, Oxford Instruments) with liquid nitrogen as cooling source. The two ends of one $(\mathrm{ET}) \mathrm{Cu}\left[\mathrm{N}(\mathrm{CN})_{2}\right]_{2}$ wire were connected into the test circuit with two electrodes using silver conductive epoxy. SourceMeter Keithley 2450 provided the electric current when Raman mapping was running. The polarized laser excitation is along wire direction ( $a$-axis). The Raman spectra were taken at a fixed temperature and by focusing on a series of points of the wire.

Computational methods: Calculations were performed with DFT+U using the PBE GGA exchange-correlation functional ${ }^{7}$ within the Quantum Espresso package ${ }^{8,9}$. A Hubbard U parameter of $5 \mathrm{eV}$ was used for the localized $3 \mathrm{~d}$ electrons of $\mathrm{Cu}$. Projector-augmented wave (PAW) pseudopotentials with scalar relativistic corrections were used. The experimental lattice parameters and structure refined from the single-crystal X-ray diffraction were used (see Table S1) for all calculations, with a $4 \times 4 \times 4$ k-point grid. $\mathrm{DFT}+\mathrm{U}-$ relaxed geometries were also computed and found to have no appreciable difference on the calculated band structure compared to that from the experimental crystal structure. The fermi surface was calculated using a 30x30x30 k-point grid and was visualized using the FermiSurfer software ${ }^{10}$.

Magnetic susceptibility and hysteresis loop measurements: Magnetic properties 
measurements were conducted on a Physical Property Measurement System (PPMS) Quantum Design EverCool II) from $5 \mathrm{~K}$ to $300 \mathrm{~K}$. $3.4 \mathrm{mg}$ as-synthesized $(\mathrm{ET}) \mathrm{Cu}\left[\mathrm{N}(\mathrm{CN})_{2}\right]_{2}$ wires were collected for the immediate magnetic properties measurements. Magnetic hysteresis loops were measured at fixed temperatures by sweeping the magnetic field from $2 \mathrm{kOe}$ to -2 kOe.

Low temperature electrical transport measurements: The low-temperature resistivity measurements of $(\mathrm{ET}) \mathrm{Cu}\left[\mathrm{N}(\mathrm{CN})_{2}\right]_{2}$ wires were taken on a Janis low temperature system with helium gas compressor (CTI-Cryogenics, Helix Technology Corp.) using a two-probe method. MG chemicals 8331D silver conductive epoxy adhesive was used for electrode contact with wire samples. 
Section 1. Sample synthesis

a

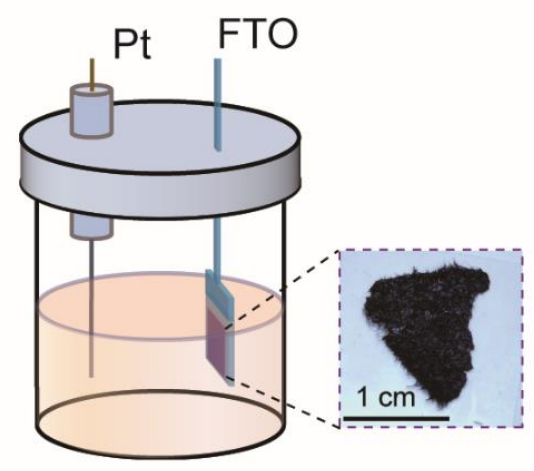

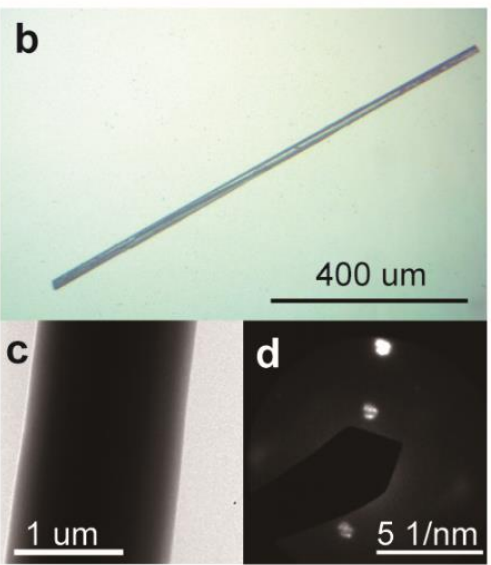

Figure S1. Electrochemical growth, morphology of (ET)Cu[N(CN) $]_{2}$. (a) Customized electrochemical reaction cell is a glass bottle with applied current flowing from FTO substrate to Pt electrode. The optical image shows the abundant $(\mathrm{ET}) \mathrm{Cu}\left[\mathrm{N}(\mathrm{CN})_{2}\right]_{2}$ wires grown on the substrate. Kinetic growth is preferred for quasi-1D wires by high current ( $\sim \mathrm{uA}$ ) compared to thermodynamic driven mechanism at a low current $(0.1 \sim 0.2 \mathrm{uA})$. (b) Optical image of a single wire shows the 1D feature. (c-d) Transmission electron microscopic image and the selected area electron diffraction pattern of a small sample wire indicate its high quality in crystallinity. 
Section 2. Structural and morphologic characterizations

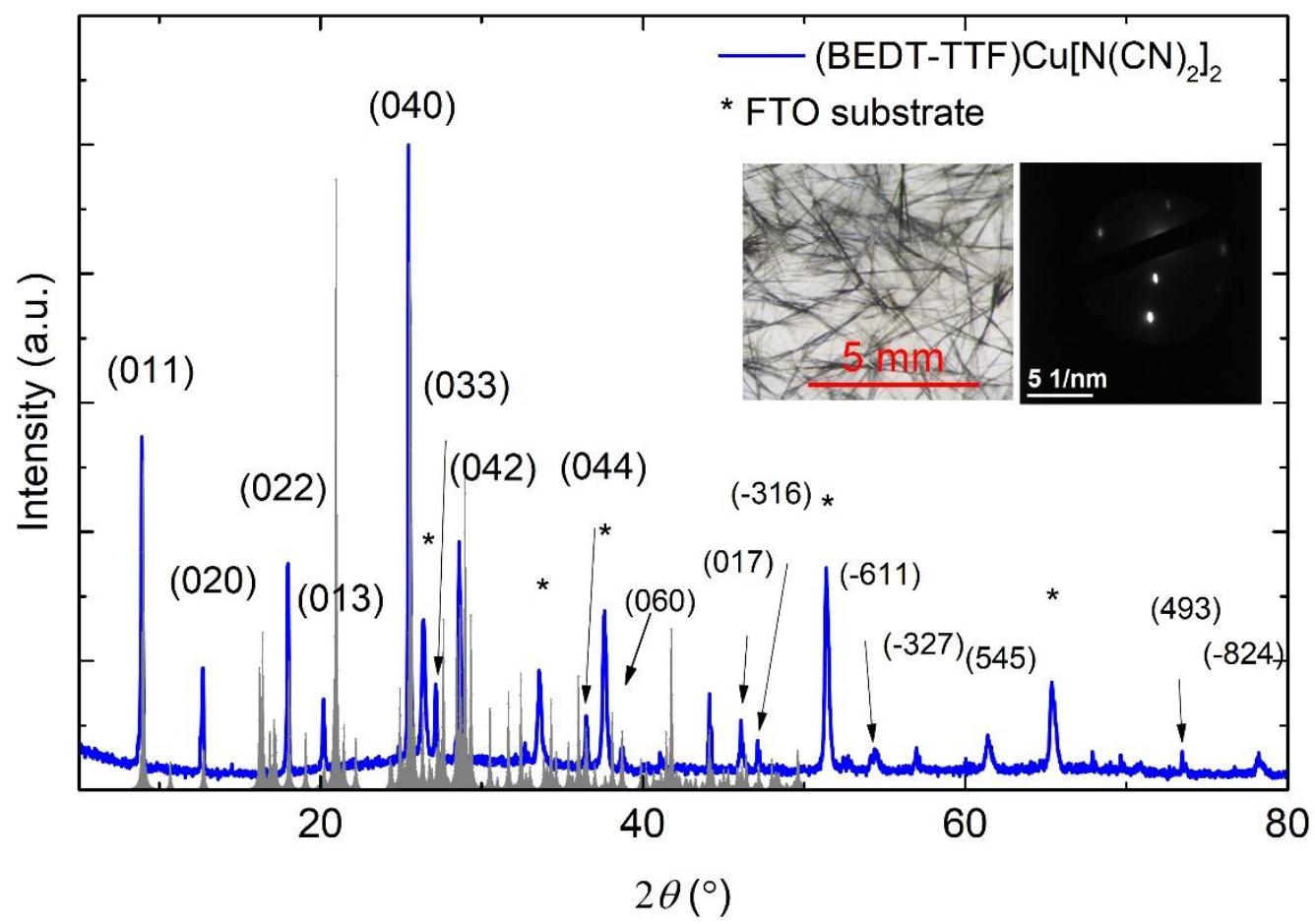

Figure S2. Powder X-ray diffraction (PXRD) pattern of vast $(\mathrm{ET}) \mathrm{Cu}\left[\mathrm{N}(\mathrm{CN})_{2}\right]_{2}$ wires on an FTO substrate (the inserted optical image). All the XRD peaks are indexed well with the refined crystal structure, except for those from the FTO substrate marked by a star symbol. The inserted optical image shows the one-dimensional (1D) wires in a length of near 1 centimeter. The selected area electronic diffraction pattern indicates the 1D feature. The grey pattern is derived from refined crystal structure. 

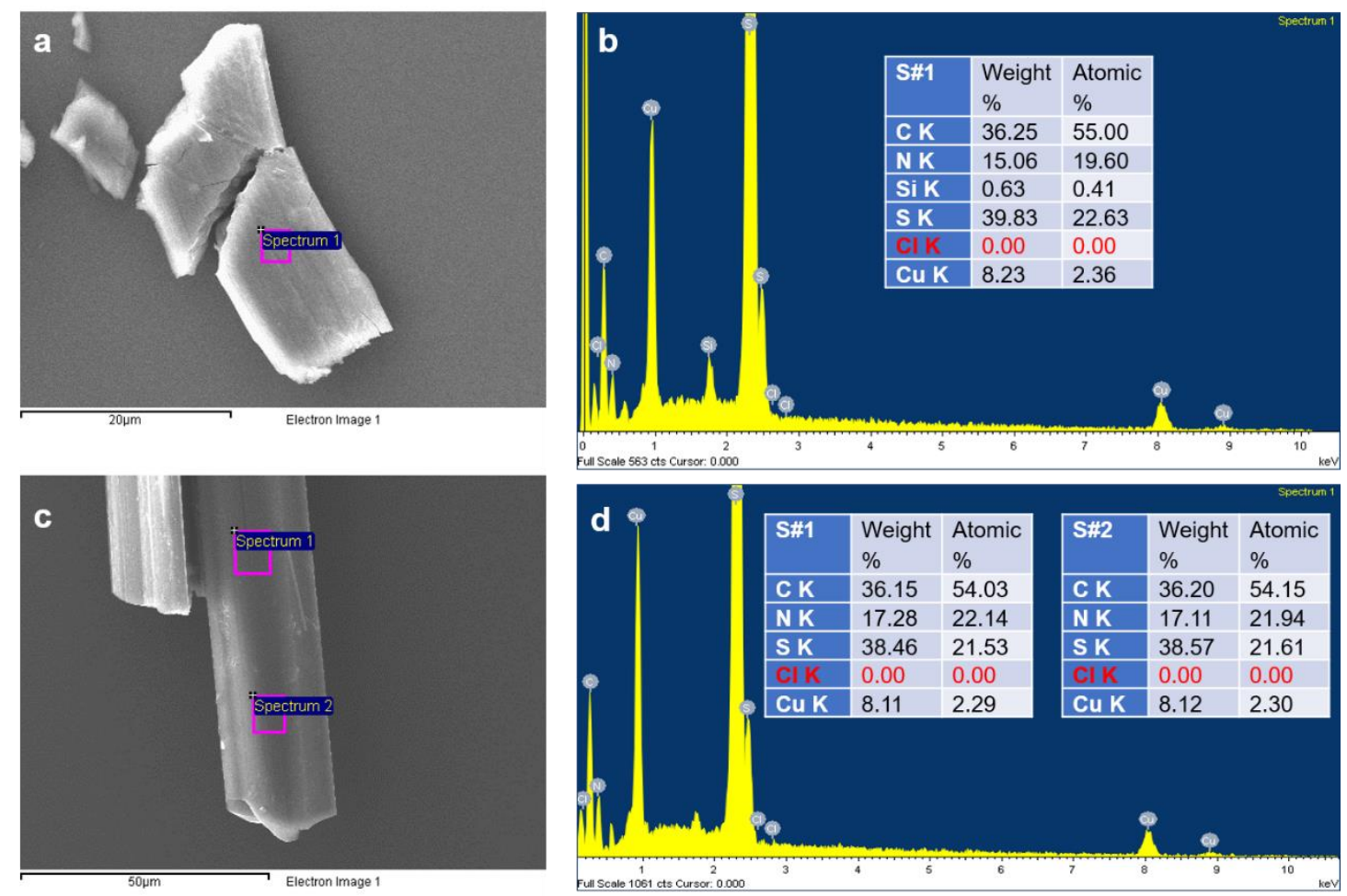

Figure S3. Energy-dispersive X-ray spectroscopy (EDS) analysis for $(\mathrm{ET}) \mathrm{Cu}\left[\mathrm{N}(\mathrm{CN})_{2}\right]_{2}$ wires. (a) SEM image of wire \#1 on a silicon substrate. (b) EDS of wire \#1 shows no detectable chlorine element. (c) SEM image of wire \#2 on a silicon substrate. (d) EDS of wire \#1 shows no detectable chlorine element on two different spots. 

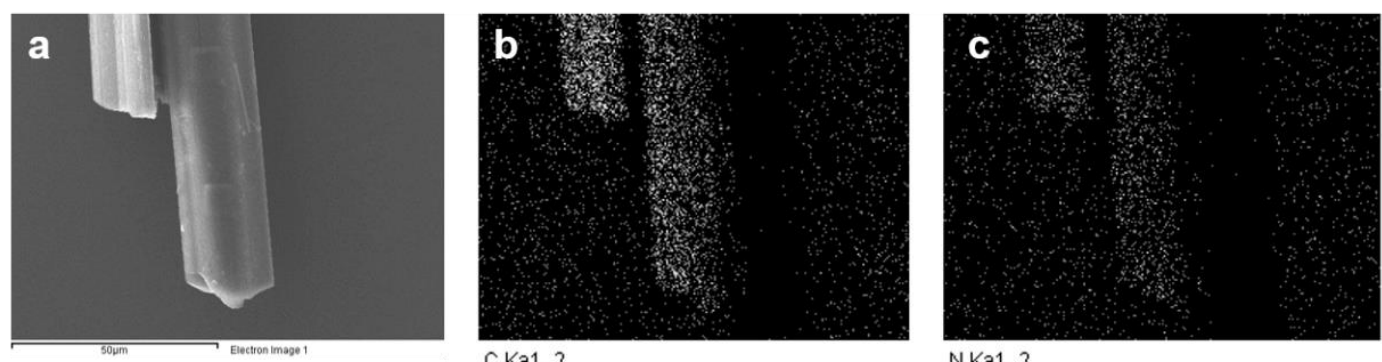

C Ka1_2

NKa1_2
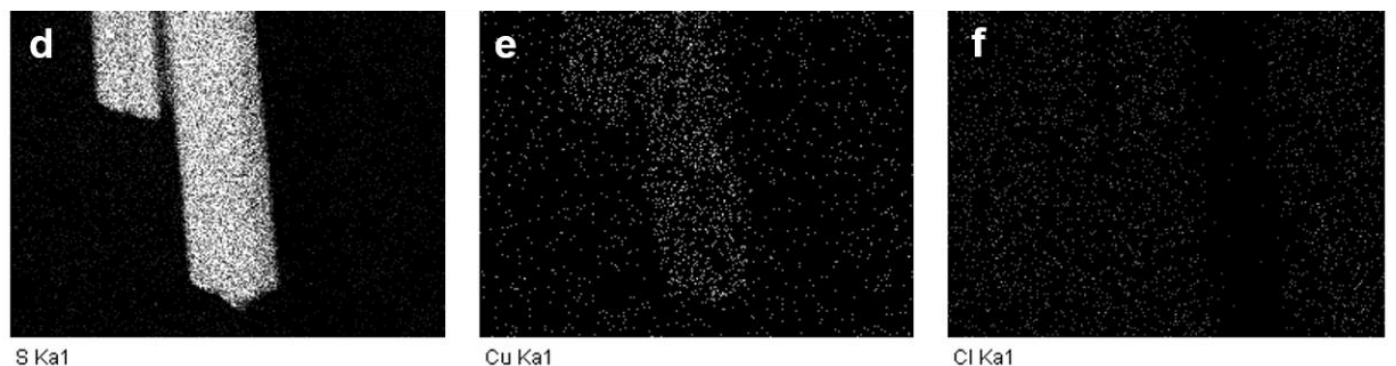

Figure S4. EDS mapping of wire \#2. (a) SEM image of wire \#2 sample. (b-e) EDS mapping results for elements $\mathrm{C}, \mathrm{N}, \mathrm{S}, \mathrm{Cu}$, respectively. f) No obvious chlorine trace can be confirmed from EDS result. 

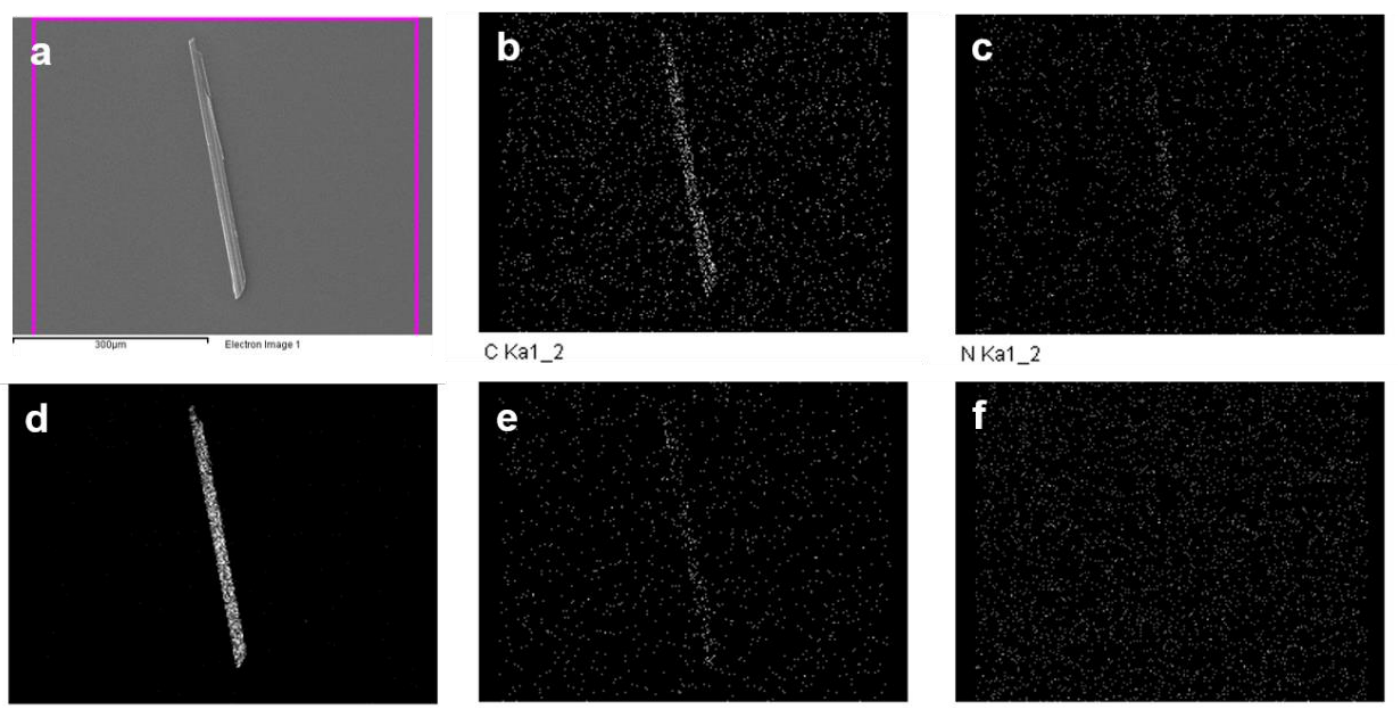

CKa1_2

NKa1_2
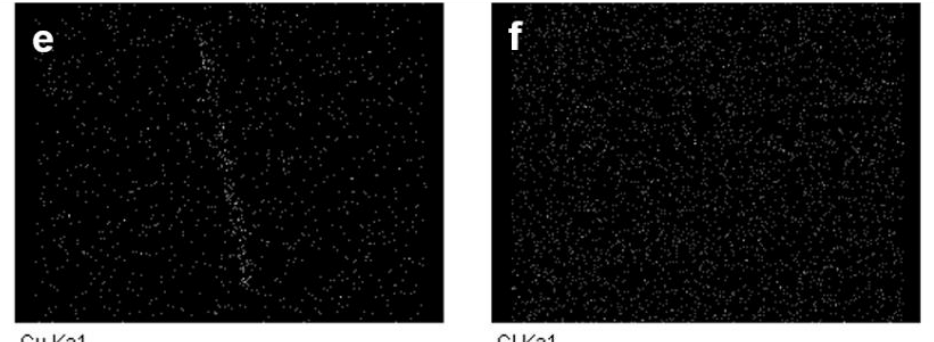

Figure S5. EDS mapping of wire \#3. (a) SEM image of wire \#3 sample. (b-e) EDS mapping results for elements $\mathrm{C}, \mathrm{S}, \mathrm{N}, \mathrm{Cu}$, respectively. f) No obvious chlorine trace can be confirmed from EDS result. 


\section{Section 3. Single Crystal X-ray Diffraction}

Purple wires of $\mathbf{1}$ crystallize in the centrosymmetric space group I2/a. It is worth noting that this crystal structure was previously reported using the space group $C 2 / c \cdot{ }^{[1]} \mathrm{We}$ acknowledge that both space groups are correct; however, in this case, the conventional space group I2/a will be used. ${ }^{[2,3]}$

a

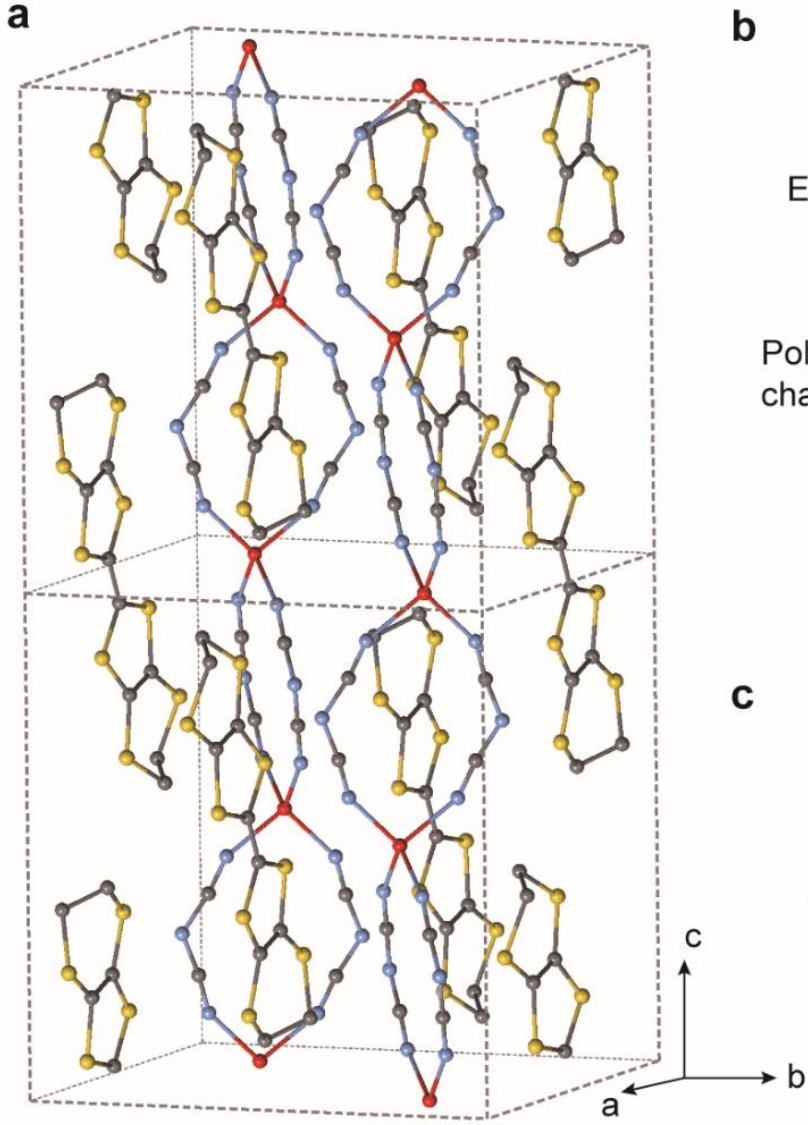

b

ET
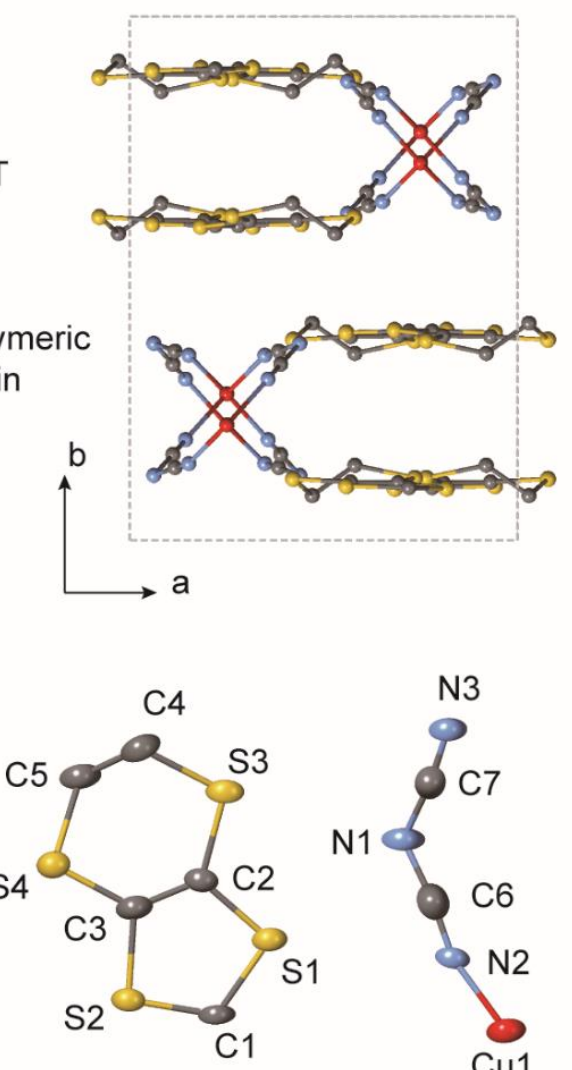

Figure S6. Crystal structure of $(\mathrm{ET}) \mathrm{Cu}\left[\mathrm{N}(\mathrm{CN})_{2}\right]_{2}$. (a) The refined crystal structure demonstrates that ET molecules and polymeric $\mathrm{Cu}\left[\mathrm{N}(\mathrm{CN})_{2}\right]_{2}$ chains are parallel stacked along $c$-axis. Only tiny overlap of sulfur atoms exists among ET molecules. (b) View of crystal structure along $c$-axis indicates ET molecules and $\mathrm{Cu}\left[\mathrm{N}(\mathrm{CN})_{2}\right]_{2}$ polymeric chains are alternatively stacking in $a b$-plane. Polymeric chain direction is perpendicular to $a b$ plane. (c) Thermal ellipsoid plot of asymmetric unit of $(\mathrm{ET}) \mathrm{Cu}\left[\mathrm{N}(\mathrm{CN})_{2}\right]_{2}$ presents the close contact between $\mathrm{ET}$ and $\mathrm{Cu}\left[\mathrm{N}(\mathrm{CN})_{2}\right]_{2}$ chain. $\mathrm{H}$ atoms are omitted for clarity. 
Table S1. Crystallographic data and structure refinement for $(\mathrm{ET}) \mathrm{Cu}\left[\mathrm{N}(\mathrm{CN})_{2}\right]_{2}$.

\begin{tabular}{|c|c|}
\hline Identification code & $(\mathrm{ET}) \mathrm{Cu}\left[\mathrm{N}(\mathrm{CN})_{2}\right]_{2}$ \\
\hline Empirical formula & $\mathrm{C}_{14} \mathrm{H}_{8} \mathrm{CuN}_{6} \mathrm{~S}_{8}$ \\
\hline Formula weight & 580.28 \\
\hline Temperature/K & 298 \\
\hline Crystal system & monoclinic \\
\hline Space group & $I 2 / \mathrm{a}$ \\
\hline $\mathrm{a} / \AA$ & $10.3099(7)$ \\
\hline $\mathrm{b} / \AA$ & $13.9369(8)$ \\
\hline $\mathrm{c} / \AA ̊$ & $13.8855(12)$ \\
\hline$\alpha /{ }^{\circ}$ & 90 \\
\hline$\beta /{ }^{\circ}$ & $93.830(2)$ \\
\hline$\gamma /{ }^{\circ}$ & 90 \\
\hline Volume $/ \AA^{3}$ & $1990.7(2)$ \\
\hline $\mathrm{Z}$ & 4 \\
\hline$\rho_{\text {calc }} \mathrm{g} / \mathrm{cm}^{3}$ & 1.936 \\
\hline$\mu / \mathrm{mm}^{-1}$ & 1.953 \\
\hline $\mathrm{F}(000)$ & 1164.0 \\
\hline Crystal size $/ \mathrm{mm}^{3}$ & $0.2 \times 0.01 \times 0.005$ \\
\hline Radiation & $\operatorname{Mo~K\alpha }(\lambda=0.71073)$ \\
\hline $2 \Theta$ range for data collection $/{ }^{\circ}$ & 4.146 to 52.8 \\
\hline Index ranges & $-12 \leq \mathrm{h} \leq 12,-17 \leq \mathrm{k} \leq 17,-17 \leq 1 \leq 17$ \\
\hline Reflections collected & 11511 \\
\hline Independent reflections & $2043\left[\mathrm{R}_{\text {int }}=0.0668, \mathrm{R}_{\text {sigma }}=0.0491\right]$ \\
\hline Data/restraints/parameters & $2043 / 0 / 132$ \\
\hline Goodness-of-fit on $\mathrm{F}^{2}$ & 1.026 \\
\hline Final $R$ indexes $[\mathrm{I}>=2 \sigma(\mathrm{I})]$ & $\mathrm{R}_{1}=0.0294, \mathrm{wR}_{2}=0.0642$ \\
\hline Final $\mathrm{R}$ indexes [all data] & $\mathrm{R}_{1}=0.0447, \mathrm{wR}_{2}=0.0689$ \\
\hline Largest diff. peak/hole / e $\AA^{-3}$ & $0.32 /-0.31$ \\
\hline
\end{tabular}

Table S2. List of short intermolecular contacts for $(\mathrm{ET}) \mathrm{Cu}\left[\mathrm{N}(\mathrm{CN})_{2}\right]_{2}$.

\begin{tabular}{cccc}
\hline \hline Atoms & $\mathrm{d}(\AA)$ & $\mathrm{r}_{\mathrm{vdw}}(\AA)$ & $\mathrm{d}-\mathrm{r}_{\mathrm{vdw}}(\AA)$ \\
\hline $\mathrm{N} 1 \cdots \mathrm{S} 1$ & $3.175(2)$ & 3.35 & -0.18 \\
$\mathrm{C} 6 \cdots \mathrm{S} 1$ & $3.201(2)$ & 3.50 & -0.30 \\
$\mathrm{~S} 1 \cdots \mathrm{S} 4^{\mathrm{i}}$ & $3.630(1)$ & 3.60 & +0.03 \\
\hline \hline
\end{tabular}

Symmetry code: (i) $1 / 2+x, 1-y, z$ 
Section 4. Magnetic susceptibility and hysteresis loop measurements
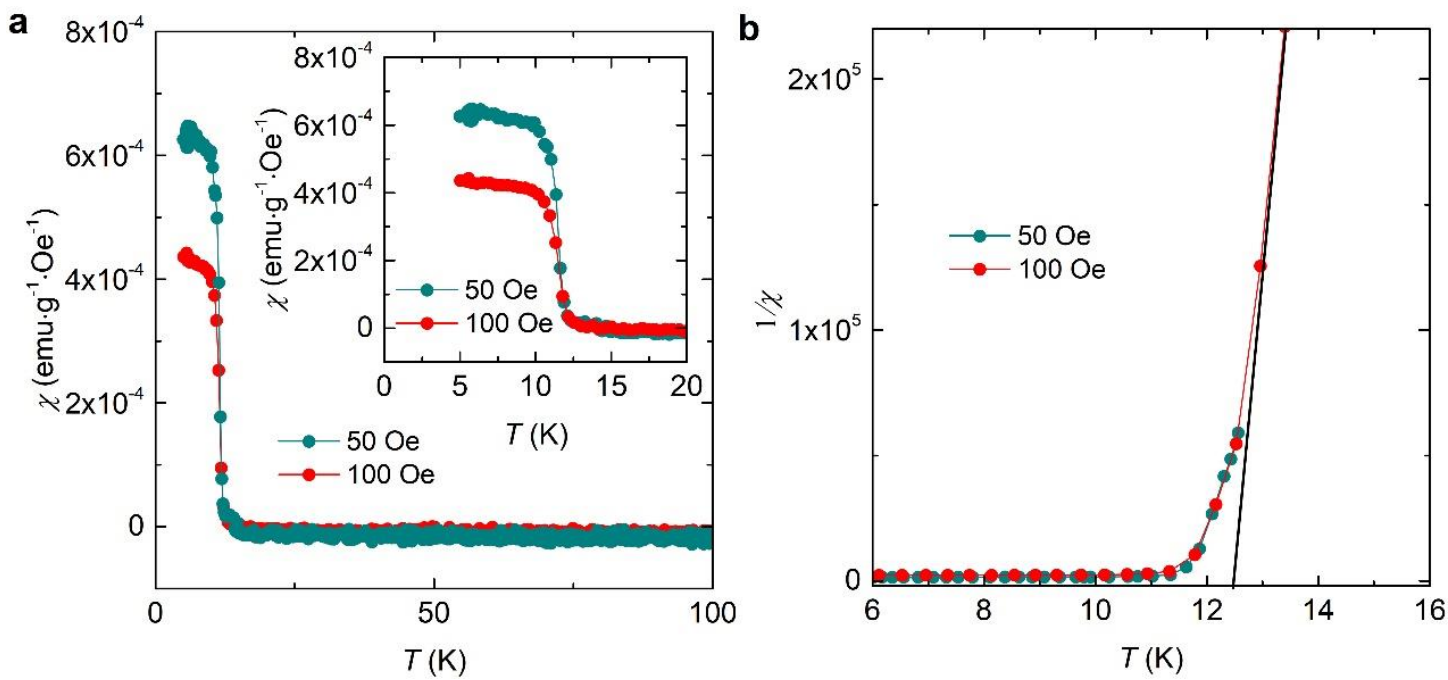

Figure S7. Magnetic properties of $(\mathrm{ET}) \mathrm{Cu}\left[\mathrm{N}(\mathrm{CN})_{2}\right]_{2}$ wires. (a) Temperature dependent magnetic susceptibility under magnetic field of 50 Oe and 100 Oe show the ferromagnetic transition. The insert figure is the enlarged one at the transition temperature. (b) The inverse magnetic susceptibility $(1 / \chi)$ presents the transition temperature is close to $13 \mathrm{~K}$. 
Table S3. Physical properties of $\kappa$-type $(\mathrm{ET})_{2} \mathrm{X}$ and $(\mathrm{ET}) \mathrm{Cu}\left[\mathrm{N}(\mathrm{CN})_{2}\right]_{2}$.

\begin{tabular}{|c|c|c|c|c|}
\hline Compounds & $T_{\mathrm{c}}$ & $E_{\mathrm{a}}$ & $\sigma$ & References \\
\hline$(\mathrm{ET}) \mathrm{Cu}\left[\mathrm{N}(\mathrm{CN})_{2}\right]_{2}$ & $13 \mathrm{~K}^{a}$ & $109.7 \mathrm{meV}$ & $1 \mathrm{mScm}^{-1}$ & This work \\
\hline$\kappa-(\mathrm{ET})_{2} \mathrm{Cu}(\mathrm{NCS})_{2}$ & $10.4 \mathrm{~K}^{b}$ & Metallic & $20 \mathrm{Scm}^{-1}$ & {$[4,5]$} \\
\hline$\kappa-(\mathrm{ET})_{2} \mathrm{Cu}\left[\mathrm{N}(\mathrm{CN})_{2}\right] \mathrm{Br}$ & $11.6 \mathrm{~K}^{b}$ & $\begin{array}{l}6 \quad \mathrm{meV} \\
(<225 \mathrm{~K})\end{array}$ & $48 \mathrm{Scm}^{-1}$ & {$[6]$} \\
\hline$\kappa-(\mathrm{ET})_{2} \mathrm{Cu}\left[\mathrm{N}(\mathrm{CN})_{2}\right] \mathrm{Cl}$ & $\begin{array}{l}45 \mathrm{~K}^{a} \\
12.5 \quad \mathrm{~K}^{b} \\
(0.3 \mathrm{kbar})\end{array}$ & $\begin{array}{l}12 \mathrm{meV} \\
(300 \mathrm{~K}) \\
52 \mathrm{meV}(50 \\
\mathrm{K})\end{array}$ & $2 \mathrm{Scm}^{-1}$ & {$[7,8]$} \\
\hline$\kappa-(\mathrm{ET})_{2} \mathrm{Cu}_{2}(\mathrm{CN})_{3}$ & $\begin{array}{l}2.8 \mathrm{~K}^{b}(1.5 \\
\text { kbar })\end{array}$ & $48 \mathrm{meV}$ & $10 \mathrm{Scm}^{-1}$ & {$[9]$} \\
\hline
\end{tabular}

$a$ : critical temperature for (anti)ferromagnetism; $b$ : critical temperature for superconductivity. 


\section{Section 5. Electrical transport properties}

Temperature dependent resistivity of $(\mathrm{ET}) \mathrm{Cu}\left[\mathrm{N}(\mathrm{CN})_{2}\right]_{2}$ at a low electric current excitation exhibits a semiconducting behavior. Three-dimensional (3D) variable range hopping (VRH) and thermal activation models are applied for data fitting of low temperature resistivity of $(\mathrm{ET}) \mathrm{Cu}\left[\mathrm{N}(\mathrm{CN})_{2}\right]_{2}$ with an increasing current excitation of $0.1 \mathrm{uA}, 1 \mathrm{uA}$, and $10 \mathrm{uA}$. Thermal activation model $\left(\rho=\rho_{0} \exp \left(T_{0} / T\right)\right)$ can provide activation energy $E_{\mathrm{a}}=k T_{0}$ ( $k$ is the Boltzmann constant, $T_{0}$ is the fitting parameter) near room temperature. 3D VRH model is suitable for the conduction mechanism at lower temperature (less than $150 \mathrm{~K}$ ).
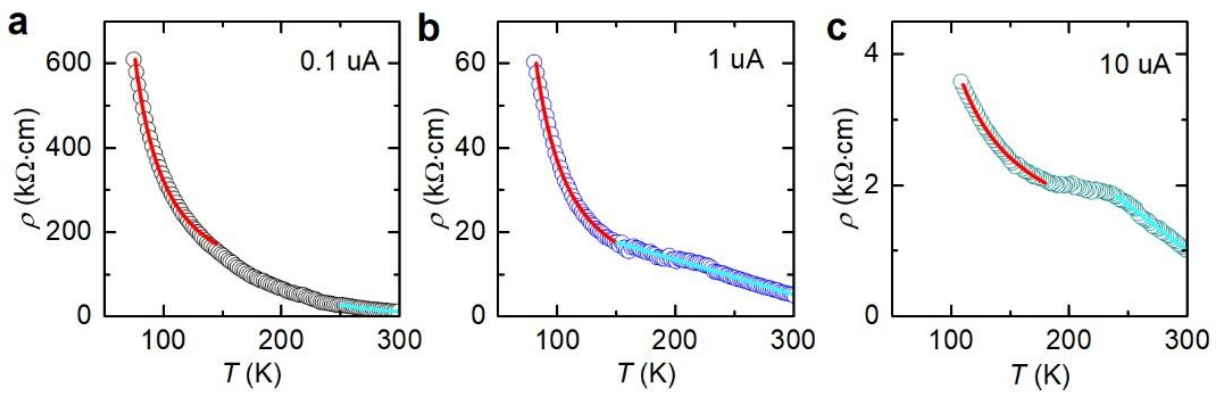

Figure S8. Temperature dependent resistivity of $(\mathrm{ET}) \mathrm{Cu}\left[\mathrm{N}(\mathrm{CN})_{2}\right]_{2}$ wire under different current excitation. 
Table S4. Fitting parameters of electron transport models for temperature dependent resistivity under different current excitations.

\begin{tabular}{|c|c|c|c|c|c|c|}
\hline \multirow{2}{*}{ Current } & \multicolumn{2}{|c|}{$\begin{array}{l}\text { 3D VRH } \\
\left(\rho=\rho_{0} \exp \left(T_{0} / T\right)^{1 / 4}\right)\end{array}$} & \multicolumn{2}{|c|}{$\begin{array}{l}\text { Thermal activation } \\
\left(\rho=\rho_{0} \exp \left(T_{0} / T\right)\right)\end{array}$} & \multicolumn{2}{|l|}{$\begin{array}{l}\text { Linear- } T \\
\left(\rho=\rho_{0}+\mathrm{A}^{*} T\right)\end{array}$} \\
\hline & Fitting range & $T_{0}$ & Fitting range & $T_{0}$ & Fitting range & A \\
\hline $0.1 \mathrm{uA}$ & $75 \sim 150 \mathrm{~K}$ & $815 \mathrm{~K}$ & $250 \sim 300 \mathrm{~K}$ & $1273 \mathrm{~K}$ & & \\
\hline $1 \mathrm{uA}$ & $80 \sim 150 \mathrm{~K}$ & $890 \mathrm{~K}$ & & & $150 \sim 300 \mathrm{~K}$ & -83 \\
\hline $10 \mathrm{uA}$ & $110 \sim 180 \mathrm{~K}$ & $627 \mathrm{~K}$ & & & $240 \sim 300 \mathrm{~K}$ & -14 \\
\hline
\end{tabular}



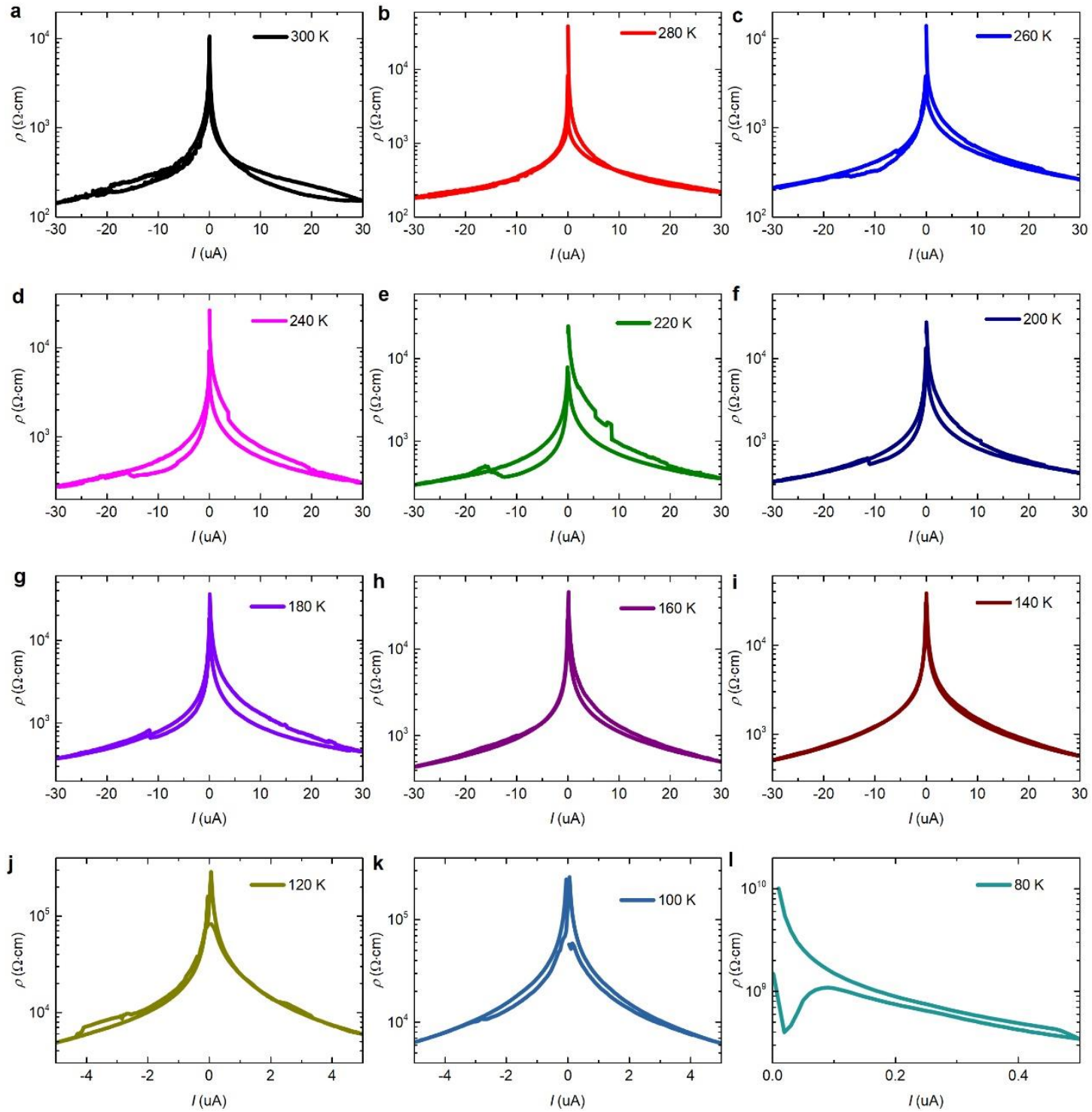

Figure S9. Resistivity versus current loops from -30 uA to $30 \mathrm{uA}$ at different fixed temperature from $300 \mathrm{~K}$ to $80 \mathrm{~K}$. 


\section{Section 6. First-principles computation}
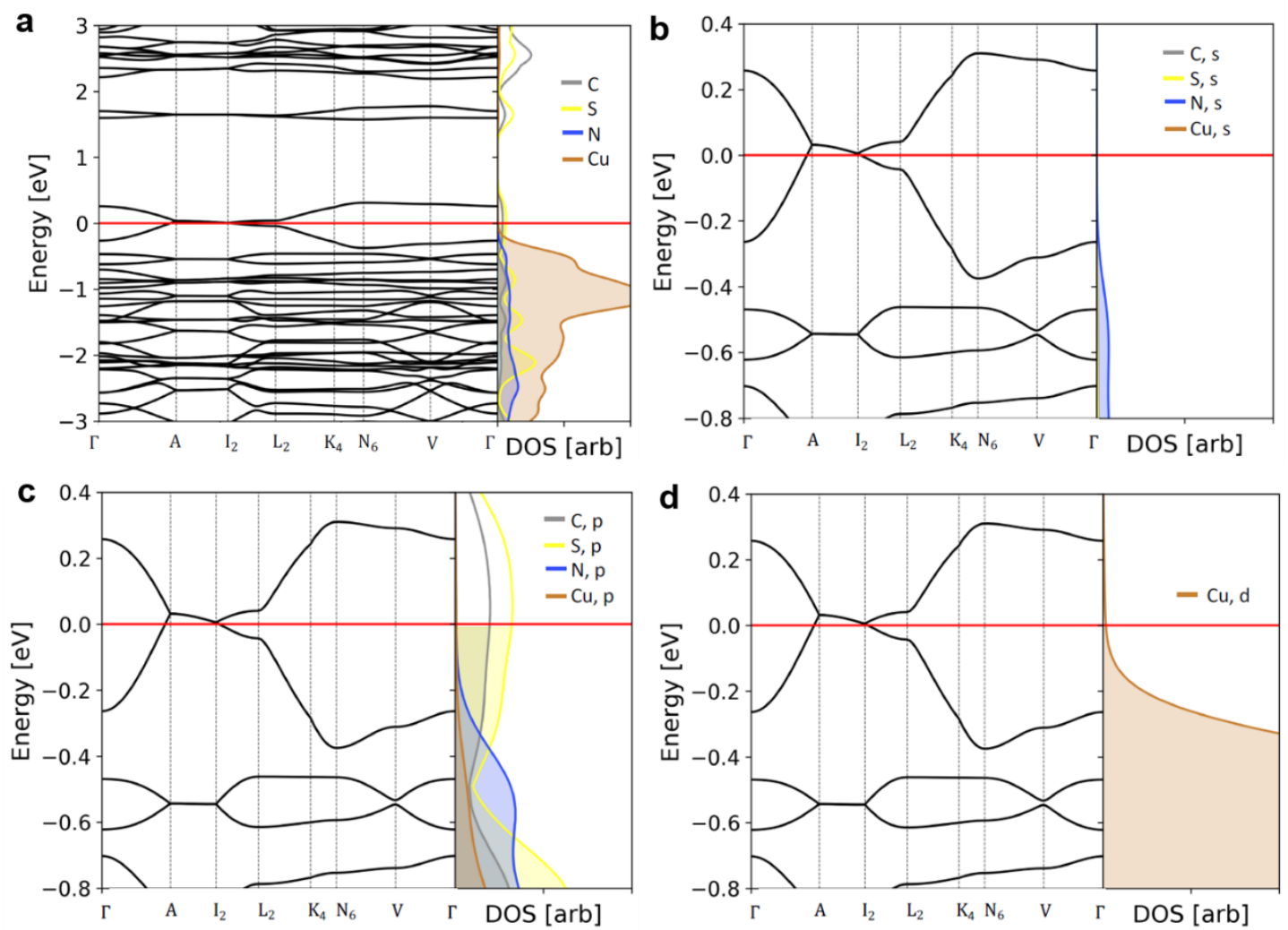

Figure S10. Projected band structure of $(\mathrm{ET}) \mathrm{Cu}\left[\mathrm{N}(\mathrm{CN})_{2}\right]_{2}$. (a) The whole band structure with density of state (DOS) of C, S, N, and $\mathrm{Cu}$ atoms. (b-d) Enlarged projected band structure near Fermi energy level with DOS of s, p, d orbitals. 
Section 7. Spectroscopic properties
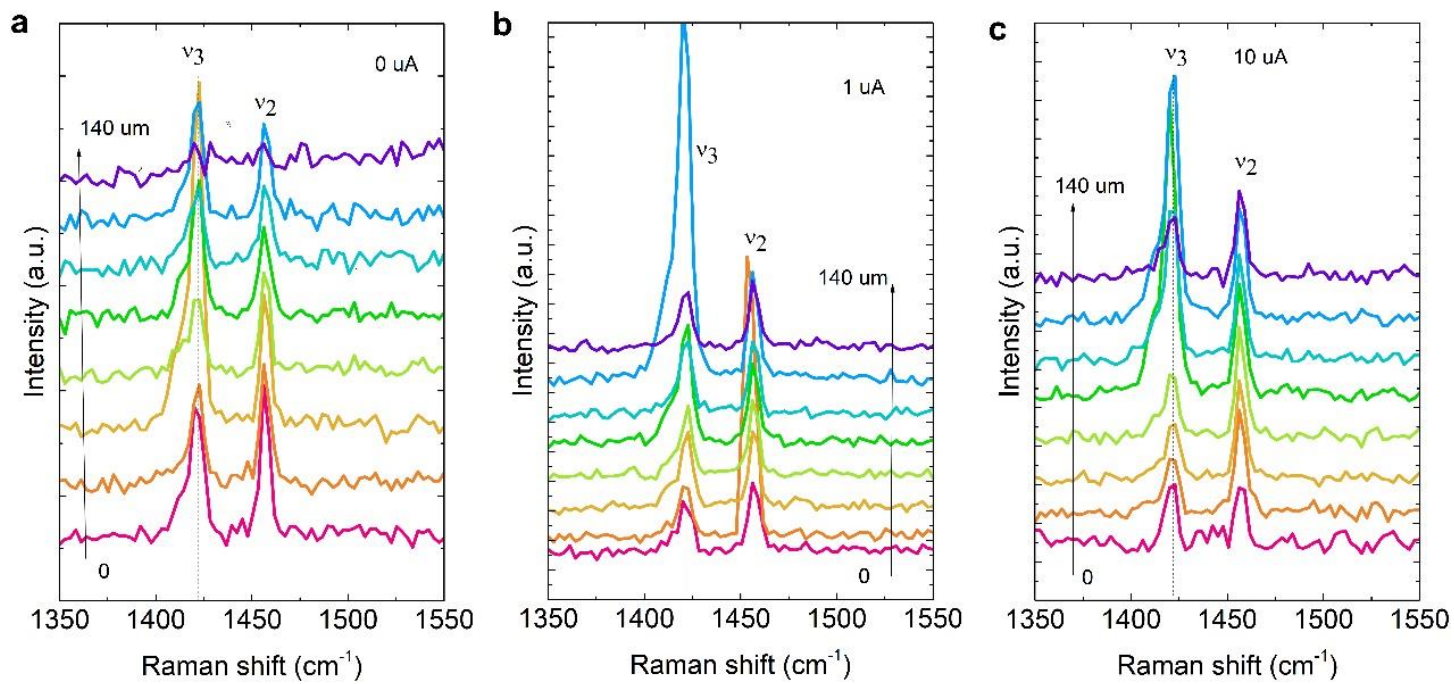

Figure S11. Raman mapping spectra along wire direction (a-axis) under different current excitation (a) $0 \mathrm{uA}$, (b) $1 \mathrm{uA}$, and (c) $10 \mathrm{uA}$. 


\section{Section 8. Diamagnetic transition}
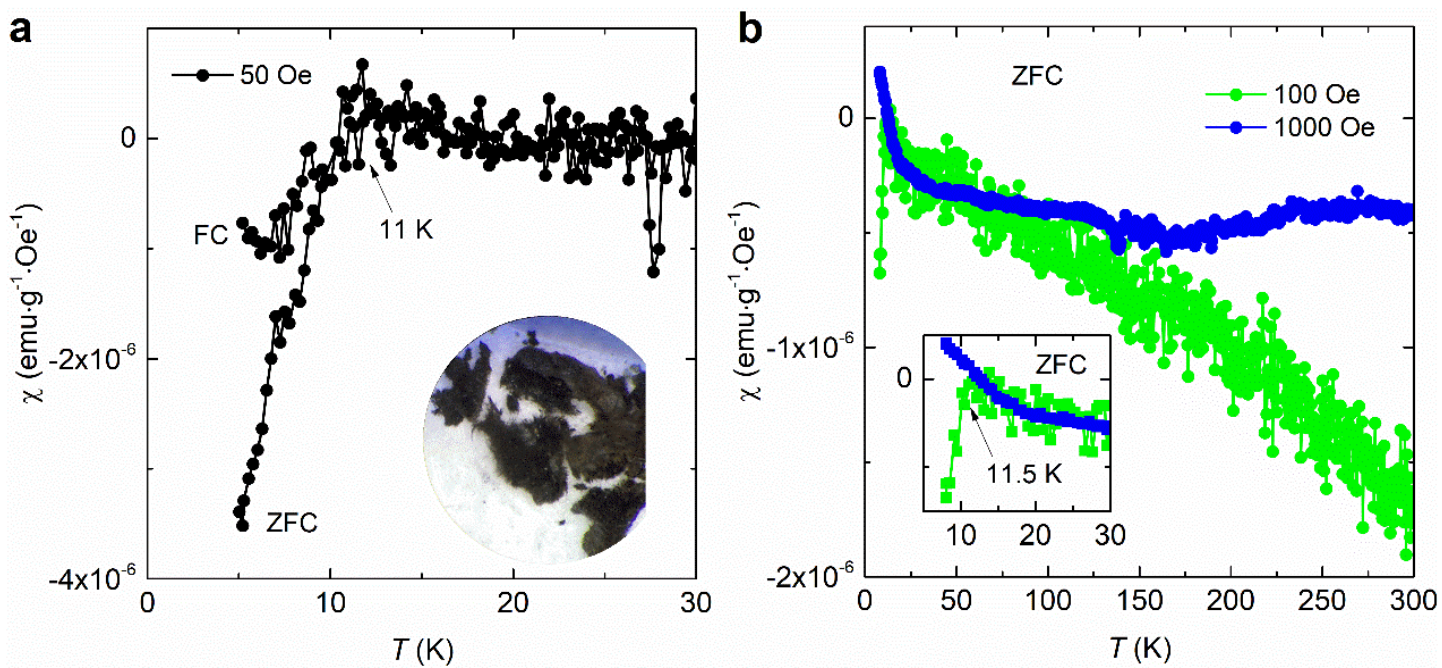

Figure S12. Diamagnetic transition in $(\mathrm{ET}) \mathrm{Cu}\left[\mathrm{N}(\mathrm{CN})_{2}\right]_{2}$ wires was observed for once. (a) Zero-field-cooled and field-cooled magnetic susceptibility show a diamagnetic transition at 11 K. (b) Temperature dependent magnetic susceptibility under 100 Oe and 1000 Oe. 


\section{Data S1. Crystallographic file}

\section{Reference}

(1) Wang, H. H.; Geiser, U.; Williams, J. M.; Carlson, K. D.; Kini, A. M.; Mason, J. M.; Perry, J. T.; Charlier, H. A.; Crouch, A. V. S., Phase selectivity in the simultaneous synthesis of the $T \mathrm{c}=12.8 \mathrm{~K}(0.3 \mathrm{kbar})$ organic superconductor $\kappa$-(BEDT-

$\mathrm{TTF})_{2} \mathrm{Cu}\left[\mathrm{N}(\mathrm{CN})_{2}\right] \mathrm{Cl}$ or the semiconductor (BEDT-TTF) $\mathrm{Cu}\left[\mathrm{N}(\mathrm{CN})_{2}\right]_{2}$. Chem. Mater. 1992, 4 (2), 247-249.

(2) Bruker APEX3, 2019.11-0: Program for Bruker CCD X-ray Diffractometer Control, Bruker AXS, Inc.: Wallingford, CT, 2013.

(3) Sheldrick, G., A short history of SHELX. Acta Crystallographica Section A 2008, 64 (1), 112-122.

(4) Dolomanov, O. V.; Bourhis, L. J.; Gildea, R. J.; Howard, J. A. K.; Puschmann, H., OLEX2: a complete structure solution, refinement and analysis program. Journal of Applied Crystallography 2009, 42 (2), 339-341.

(5) Sheldrick, G., SHELXT - Integrated space-group and crystal-structure determination. Acta Crystallographica Section A 2015, 71 (1), 3-8.

(6) Sheldrick, G., Crystal structure refinement with SHELXL. Acta Crystallographica Section C 2015, 71 (1), 3-8.

(7) Perdew, J. P.; Burke, K.; Ernzerhof, M., Generalized Gradient Approximation Made Simple. Phys. Rev. Lett. 1996, 77 (18), 3865-3868.

(8) Giannozzi, P.; Baroni, S.; Bonini, N.; Calandra, M.; Car, R.; Cavazzoni, C.; Ceresoli, D.; Chiarotti, G. L.; Cococcioni, M.; Dabo, I.; Dal Corso, A.; de Gironcoli, S.; Fabris, S.; Fratesi, G.; Gebauer, R.; Gerstmann, U.; Gougoussis, C.; Kokalj, A.; Lazzeri, M.; Martin-Samos, L.; Marzari, N.; Mauri, F.; Mazzarello, R.; Paolini, S.; $\quad$ Pasquarello, A.; Paulatto, L.; Sbraccia, C.; Scandolo, S.; Sclauzero, G.; Seitsonen, A. P.; Smogunov, A.; Umari, P.; Wentzcovitch, R. M., QUANTUM ESPRESSO: a modular and open-source software project for quantum simulations of materials. J. Phys.: Condens. Matter 2009, 21 (39), 395502.

(9) Giannozzi, P.; Andreussi, O.; Brumme, T.; Bunau, O.; Buongiorno Nardelli, M.; Calandra, M.; Car, R.; Cavazzoni, C.; Ceresoli, D.; Cococcioni, M.; Colonna, N.; Carnimeo, I.; Dal Corso, A.; de Gironcoli, S.; Delugas, P.; DiStasio, R. A.; Ferretti, A.; Floris, A.; Fratesi, G.; Fugallo, G.; Gebauer, R.; Gerstmann, U.; Giustino, F.; Gorni, T.; Jia, J.; Kawamura, M.; Ko, H. Y.; Kokalj, A.; Kucukbenli, E.; Lazzeri, M.; Marsili, M.; Marzari, N.; Mauri, F.; Nguyen, N. L.; Nguyen, H. V.; Otero-dela-Roza, A.; Paulatto, L.; Ponce, S.; Rocca, D.; Sabatini, R.; Santra, B.; Schlipf, M.; Seitsonen, A. P.; Smogunov, A.; Timrov, I.; Thonhauser, T.; Umari, P.; Vast, N.; Wu, X.; Baroni, S., Advanced capabilities for materials modelling with Quantum ESPRESSO. J. Phys.: Condens. Matter 2017, 29 (46), 465901.

(10) Kawamura, M., FermiSurfer: Fermi-surface viewer providing multiple representation schemes. Comput. Phys. Commun. 2019, 239, 197-203. 